\title{
Cardio-metabolic risk estimation at population setting, an approach to pragmatic health screening
}

\author{
Hussain HY1*, Abady $\mathrm{KL}^{2}$, Salim NM${ }^{3}$, Aguilar $\mathrm{KKD}^{4}$ and Tuffaha $\mathbf{M}^{5}$ \\ ${ }^{1}$ Research Studies and data analysis dept, Dubai Health Authority, United Arab Emirates \\ ${ }^{2}$ Public Health Dept protection, Dubai Health Authority, United Arab Emirates \\ ${ }^{3}$ Dubai hospital, Dubai Health Authority, Dubai, United Arab Emirates \\ ${ }^{4}$ Latifa Hospital, Dubai Health Authority, United Arab Emirates \\ ${ }^{5}$ Amana Health Care Medical and Rehabilitation Hospital, Abu Dhabi, United Arab Emirates
}

Research Article

Volume 4 Issue 2

Received Date: April 09, 2020

Published Date: May 05, 2020

DOI: $10.23880 /$ eij-16000144

*Corresponding author: Hussain HY, Dubai Health Authority, Research Studies and data analysis dept, Dubai, United Arab Emirates, Tel: 00971502608873; Email: Hyhussain@dha.gov.ae

\section{Abstract}

Background: Unhealthy behaviors include poor dietary habits and a sedentary lifestyle. There are two types of Diabetes Mellitus with a prevalence ranging from 1\% in rural Uganda to $12 \%$ in urban Kenya. Overweight and obesity have been found to be modifiable risk factors for cardio metabolic and other chronic diseases such as hypertension, diabetes and dyslipidemia. Objectives: To study the baseline prevalence of cardio metabolic risk factors, Sociodemographic characteristic and distribution of cardio metabolic risk factors among the population in Dubai.

Materials \& Methods: A secondary data of household health surveys was carried out in Dubai in 2019. This is done in cooperation with Dubai Health Authority and Dubai Statistics Center. Sampling, survey design, data collection, data weighting and data analysis has been carried out based on an advanced statistical and epidemiological applications to ensure the validity and reliability of the survey outcomes. More than 400 health indicators were covered by this survey, among which includes health behavior factors (e.g. physical activity, tobacco use, adequate consumption of fruits \& vegetable and obesity) as well as diabetes mellitus and hypertension. Field work was done by 100 surveyors including 25 nurses, who were well trained and continued to work for 40 days and covered visits up to 2400 households (families). In the said survey there were about 10000 individuals (both males and females) of different nationalities and of all ages (children, adults, elderly and labors). Strict operational definitions of variables were used according to WHO. Variables measured were blood pressure, blood sampling, BMI, waist circumference and abdominal circumference and standard questionnaire by WHO and World Bank was adopted for data collection.

Results: The present study showed that only $19.90 \%$ of the total population was classified as a physically active group and $80.10 \%$ were classified as a group with a non-adequate practice of physical activities. The current study revealed that $20.80 \%$ and $31.80 \%$ of the target population were identified as overweight with BMI $25-29 \mathrm{~kg} / \mathrm{m} 2$. The current study results reflect that only $36.3 \%$ of the Dubai Population consumes adequate fruits and vegetables in their daily practice while $63.6 \%$ were categorized as consumers with inadequate food and vegetable intake. It has been shown that $17.7 \%$ of total Dubai population was diagnosed with Diabetes having an HBA1c of more than $6.5 \%$. About $2.1 \%$ of which were newly diagnosed as Diabetes and $11.6 \%$ were self-reported individuals having diabetes mellitus. Furthermore, about $32 \%$ of the total population was 


\section{Epidemiology International Journal}

diagnosed as hypertensive group. The present study revealed that $15.8 \%$ of the total population were recognized as currently active smokers as seen in table.

Conclusion: The study revealed a significant rise in the current trend of cardio metabolic risks among the Dubai Population, which is going to steer a remarkable medium and long-term burdens (Mortalities, morbidity and complications), quality and productivity of life as well as care costs.

Keywords: Cardio Metabolic Risk; Dubai; Burdens

Abbreviations: CVDs: Cardiovascular diseases; NCDs: non-communicable diseases; IDF: International Diabetes Federation.

\section{Baseline Prevalence Cardio-Metabolic Risk Assessment at Population Setting, from Temporal Time Trend to Long-Term Burdens, Experience of Dubai}

\section{Introduction}

Cardiovascular diseases (CVDs) are the leading cause of death [1] worldwide. They are emerging as a significant public health phenomenon among the developing countries [2] which is witnessed through epidemiological transitions from communicable to non-communicable diseases (NCDs). Such transition has been closely linked to increase in modern lifestyle, behavioral risk modifications, urbanization and rural-urban transitions, which has led to an unhealthy living including but not restricting to poor dietary habits low physical activity and an increasing sedentary life pattern $[2,3]$.

As per the International Diabetes Federation (IDF), about 200 million people in the global level are estimated to have type 2 Diabetes Mellitus [4] with the prevalence rate ranging from $1 \%$ to $20 \%$ from different countries [5,6]. Obesity as well as overweight has been proved to be a modifiable factor threatens cardio metabolic and other NCD diseases [7] like hypertension [8] diabetes [9] and hyperlipidemia [10]. The increasing evidences that has been generated recently by many studies and researchers which was identified as central obesity, hyperlipidemia (hypertriglyceridemia and low levels of high-density lipoprotein cholesterol (HDL-C), impaired glucose tolerance and elevated blood pressure were cardio metabolic risk factors [11].

It is concluded that with the enormous and fast economic development, dramatic changes in life patterns (e.g. reduced physical activity and higher calorie and cholesterol intake), the frequencies and profiles of four signatures such as obesity, diabetes, hypertension and dyslipidemia have changed even more rapidly [12-15]. Having said that, the ongoing deterioration of these cardio metabolic risk factors worldwide may greatly contribute to the surge of cardio metabolic health, more understanding of such factors shall be quite effective in the assessment and management operations. Even up to now, little is understood about the time trend of these risk factors [16-19].

\section{Objectives}

1. To study baseline prevalence of cardio metabolic risk factors among Dubai Population, to study sociodemographic characteristics and distribution of cardio metabolic risk factors amongDubai population

2. To study the baseline prevalence of cardio metabolic risk factors, sociodemographic characteristic and distribution of cardio metabolic risk factors among the population in Dubai.

\section{Methodology}

The study design was comprehensive household health surveys carried out in Dubai in 2019. The survey was conducted by cooperation of Dubai Health Authority and Dubai Statistics Center. Multistage stratified cluster sampling was applied for sample selection. Data collection data was carried out by 75 well trained surveyor and 25 well trained nurses through direct visit to households under direct supervision of survey conduction expert from Dubai statics center along 50 days, Data auditing, cleaning, weighting and data analysis was carried out by expert statisticians and data analysts advanced statistical and epidemiological applications (Epi info, SPSS21, and Stata 12software) to ensure the validity and reliability of the survey outcomes. More than 400 health indicators were obtained by this survey, among which includes health behavior factors (e.g. physical activity, tobacco use, adequate consumption of fruits \& vegetable and obesity) as well as diabetes mellitus and hypertension.

Up to 2400 households (families) were visited equal to 10000 individuals. (Both males and females) of different nationalities (UAE nationality and Non UAE nationalities) and of all ages (children 0-17 years, adults18-59 years, elderly 60+ and labors). Current study is handling the data and the results 


\section{Epidemiology International Journal}

relevant to adult group (18-59 years) which consist (2245) individual plus $60+$ cohorts. Strict operational definitions of variables were used according to WHO. Variables measured were (3) times blood pressure measurements both systolic and diastolic by high sensitive and well calibrated electronic sphygmomanometers, blood sampling (HBA1c), BMI, waist circumference and abdominal circumference. Standard questionnaire of 20 pages (1400 questions) based on WHO and World Bank for household health surveys was utilized data collection tool, which covered (Health behaviors and life style, NCDs, Immunizations, Health expenditure, Mental Health, Health services, Elderly health).

\section{Results}

Figure 1 showed that only $20 \%$ of total population was classified as physically active group and $80 \%$ In addition, data was classified as adequate and non-adequate practicing of physical activities. While Figure 2 revealed that about $21 \%$ and $32 \%$ were categories as overweight BMI of $25-29$ $\mathrm{kg} / \mathrm{m}^{2}$. The current study results reflected that only $36 \%$ of Dubai Population consuming adequate fruits and vegetables in their daily practice while $64 \%$ were categorized as no adequate consumers as shown in Figure 3. It has been shown that $17.7 \%$ of total Dubai population was diagnosed as diabetic HBA1c more than 6.5. While 2.1\% newly diagnosed as Diabetes and $11.6 \%$ were self-reported of diabetes mellitus, about $32 \%$ of total population were diagnosed as hypertensive group as reflected by Figure $4 \& 5$. Table 1 showed that $15.8 \%$ of total population was recognized as current active smokers.

\begin{tabular}{|c|c|c|c|c|c|}
\hline & Current Smokers & Ex-Smokers & Never Smokers & $\begin{array}{l}\text { Mean age when } \\
\text { starts smoking }\end{array}$ & $\begin{array}{c}\text { No. of } \\
\text { respondents }\end{array}$ \\
\hline \multicolumn{6}{|l|}{ Age } \\
\hline $18-24$ & $12.90 \%$ & $0.90 \%$ & $86.20 \%$ & $21.6( \pm 1.0)$ & 224 \\
\hline $25-44$ & $17.40 \%$ & $2.40 \%$ & $80.20 \%$ & $21.3( \pm 5.2)$ & 1341 \\
\hline $45-59$ & $11.20 \%$ & $2.50 \%$ & $86.30 \%$ & $20.5( \pm 7.7)$ & 434 \\
\hline $60+$ & $17.50 \%$ & $1.70 \%$ & $80.90 \%$ & $25.5( \pm 5.8)$ & 246 \\
\hline \multicolumn{6}{|l|}{ Gender } \\
\hline Female & $7.90 \%$ & $1.30 \%$ & $90.80 \%$ & $22.8( \pm 3.7)$ & 962 \\
\hline Male & $20.90 \%$ & $2.50 \%$ & $76.60 \%$ & $21.2( \pm 5.8)$ & 1283 \\
\hline \multicolumn{6}{|l|}{ Nationality } \\
\hline UAE-nationals & $14.30 \%$ & $2.70 \%$ & $83.00 \%$ & $19.5( \pm 5.4)$ & 844 \\
\hline Non-UAE-nationals & $16.00 \%$ & $2.20 \%$ & $81.80 \%$ & $21.4( \pm 5.7)$ & 1401 \\
\hline Total & $15.80 \%$ & $2.20 \%$ & $82.00 \%$ & $21.3( \pm 5.4)$ & 2245 \\
\hline
\end{tabular}

Table 1: Distribution of respondents by prevalence of smoking and average age when starts smoking, according to background characteristics, DHHS, 2019.

\section{Physical activity among Dubai population 2019}

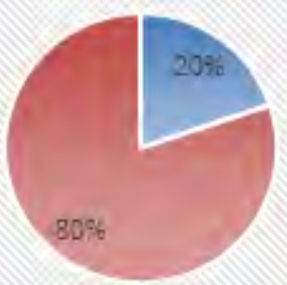

m Adequate physical Activity among Dubai Population

- Non- Adequare physical Activity among Dubai Population

Figure 1: Physical activity among Dubai Population, 2019. 


\section{Obesity and overweight among Dubai population 2019
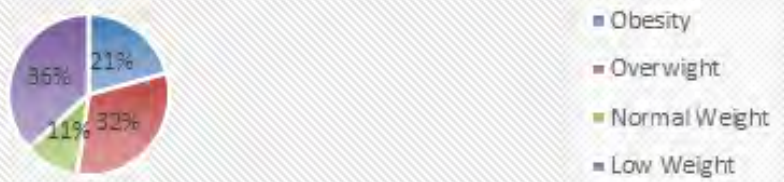

Figure 2: Obesity and overweigh among Dubai Population, 2019.

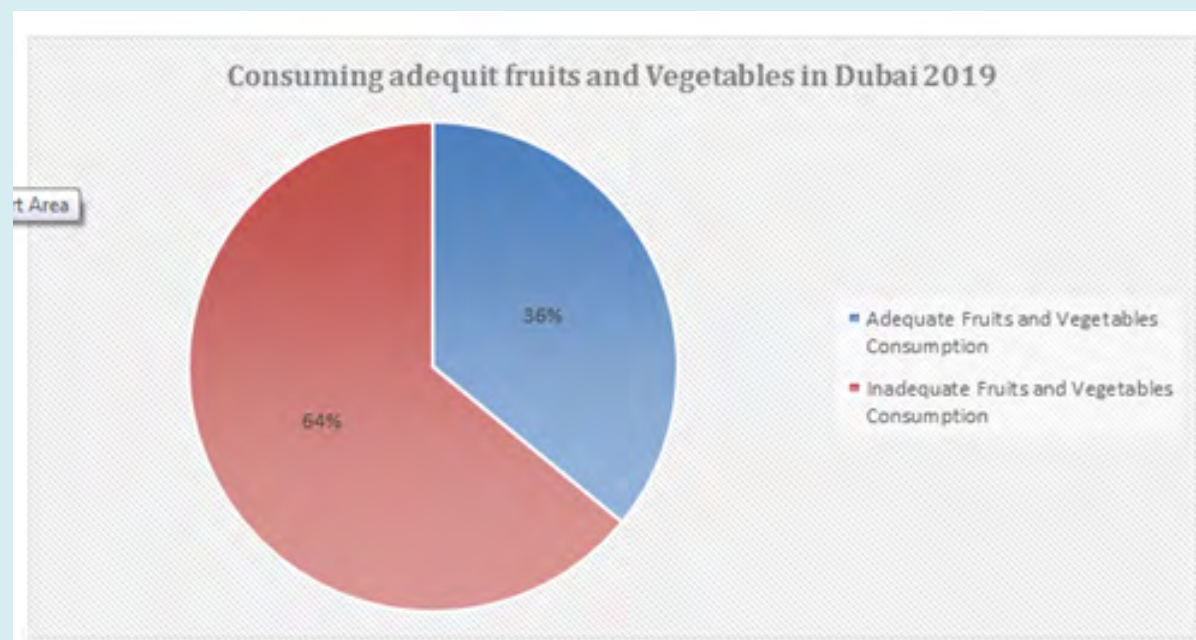

Figure 3: Consuming Healthy Diet among Dubai Population, 2019.

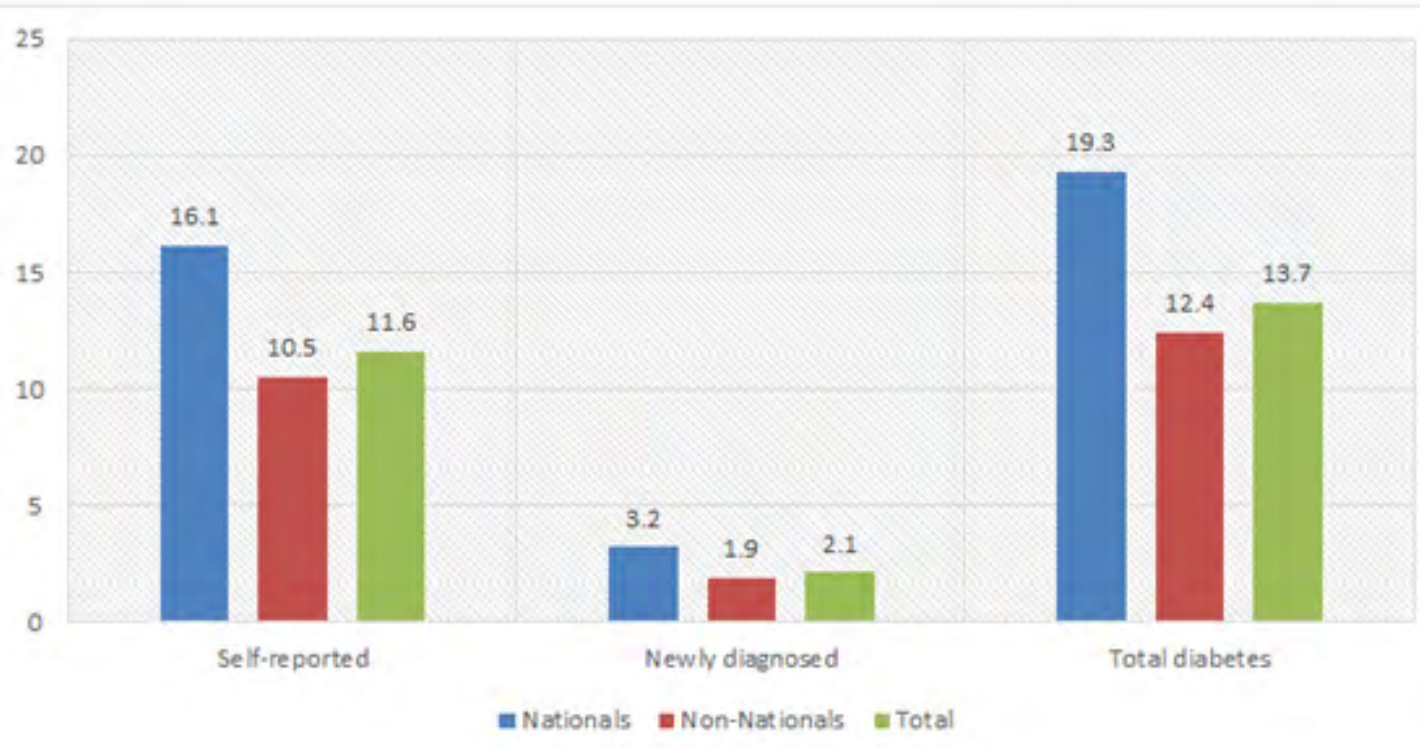

Figure 4: Distribution of Diabetes (Way of Diagnosis) among Adult Participants by Nationality. 


\section{Epidemiology International Journal}

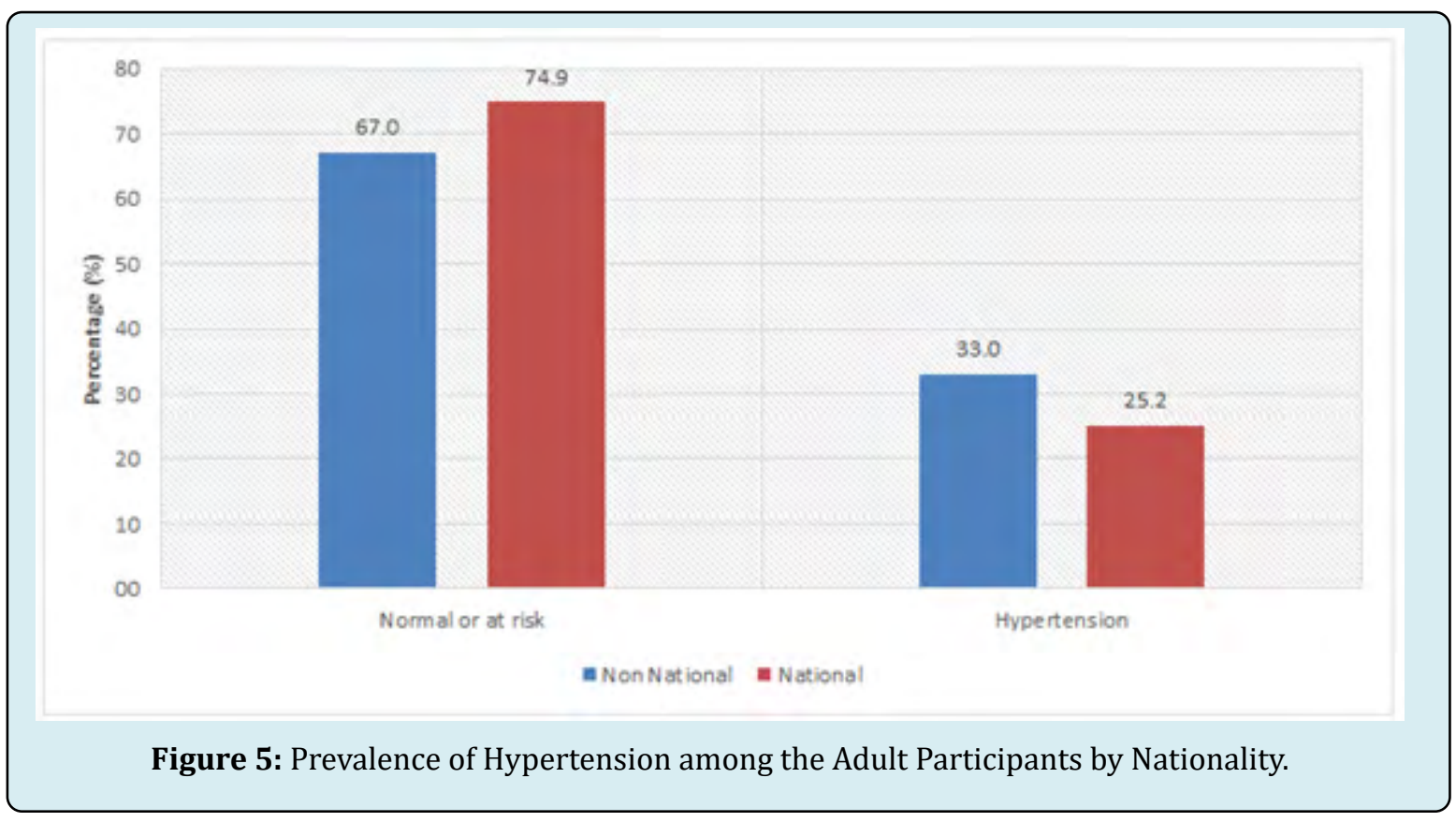

\section{Discussion}

The current study showed a significant increase of multiple cardio metabolic risks among Dubai population, which will put many burdens on the health system, cost of care, individual's quality of life and productivity. The overall prevalence of diabetes was $13.7 \%$ were among high figures worldwide, and $16.2 \%$ were among the pre-diabetics and $2.1 \%$ of the respondents did not know they were diabetics (newly diagnosed) as they were newly diagnosed through the blood sample test during the survey, such figures are alarming to develop more interventions at different levels, that findings put more population at excessive need of care and overweigh the cost capacity of the health care system as well as increase NCDs mortality.

The percent of the pre-diabetics doubled in males who consist of $20.5 \%$ than females with only $11.9 \%$. The burden of diabetes was more in UAE nationals (19.3\%) than nonUAE nationals (12.4\%). In addition, more UAE nationals (17.1\%) were detected to have a prediabetes state than nonUAE nationals (12\%). Regarding hypertension; about 32.5\% of adult individuals surveyed in Dubai have hypertension. Amongst them, 24.1\% did not know they have the condition (potential undiagnosed hypertension) and was diagnosed by the survey. Furthermore, about $32.8 \%$ of the surveyed adults were pre-hypertensive. Variations by gender were obvious, as hypertension was more currently prevalent in males (38.3\%) than in females $(16.7 \%)$. The burden of hypertension was slightly higher among non-UAE nationals $(33 \%)$ than in UAEnationals (25.2\%). Hypertension increased considerably by age, from $22.9 \%$ among respondents in the $18-24$ age group to $56.5 \%$ among those in the $60+$ age group. The current study revealed that $15.8 \%$ of the surveyed samples were currently smokers, among them $11.5 \%$ were regular smokers; while the $4.3 \%$ were irregular smokers (they were not smoke daily). Almost one third of (36.1\%) respondents reported that they ate enough fruits and vegetables on a typical day, with the vast majority of surveyed population stating that they do not eat five servings per day.

The healthy eating habit seems to be affected largely by age, whereas only $29.7 \%$ of the younger respondents reported eating sufficient amount of fruits and vegetables, compared to almost half $(45.9 \%)$ of the elder age group $(60+)$. No differences were detected either by gender or nationality among the respondents in eating sufficient fruits and vegetables on a typical day. Only one out of five respondents (19.9\%) has sufficient exercise over the course of a typical week. The percentage of males who attained the target of sufficient exercise was similar to those of females.

Obviously, the youngest age group (18-24) were the most to achieve the target compared to other groups. Adult overweight, including obesity (BMI of $25 \mathrm{~kg} / \mathrm{m}^{2}$ or greater) prevalence reached $62.1 \%$ among the sampled population of DHHS 2019. This illustrates that two in every ten of the adult participants in this DHHS were considered obese (20.8\%), with the largest proportion of the population overweight at $41.3 \%$. Only $35.8 \%$ of adults were in the healthy weight category. These findings are in similar with other studies findings in China 2010 which concluded that during the last decade (10 Years), the prevalence of obesity and overweight reflected an ongoing upward trend [20]. It shows that the 


\section{Epidemiology International Journal}

worldwide ranking of obese population in China has stepped up to the second position in 2014 [21].

Throughout the past decades, the Chinese population has revealed more sedentary life patterns, particularly for the working staff or retiree in the agency [22]. Hypertension was reported to be the most prominent cardio metabolic risk factors groups in Chinese population [23,24]. During 1988 to 2008, there was an up going trend of prevalence of hypertension in Americans older than 40 years [25] and nevertheless, the prevalence of hypertension in Germany [26], Japan [27] Italy [28] and Korea [29] have decreased. On the contrary to the present study in Dubai, there is a significant decline in the prevalence of the trend for hypertension among Chinese adults in 2017 compared to 2008. Despite the improvement of the socioeconomic status in Dubai which may contribute positively in enhancing awareness and control of hypertension, people would have better compliance to medical treatment. In China the cardiometabolic risk assessment survey, awareness, treatment and control rates as well as the treatment control rate of hypertension have improved much as compared to the previous decades which led to a better prevalence of these risks [30].

\section{Conclusion}

The study revealed a significant rise in the current trend of cardio metabolic risks among the Dubai population, which is going to steer a remarkable medium and longterm burdens, Mortalities, morbidity and complications. Moreover, these risks affect quality and productivity of life as well as care costs.

\section{Recommendation}

Risk rating and risk management at population level needs to be addressed carefully. National framework taskforces should be considered as a priority for the current health system, implementing preventive interventions, health promotions, social mobilizations, health legislations and advocacy movement.

\section{References}

1. World Health Organization (WHO) (2016) Fact sheet: Cardiovascular diseases (CVDs).

2. Oladapo O, Salako L, Sodiq O, Shoyinka K, Adedapo $\mathrm{K}$, et al. (2010) A prevalence of cardio metabolic risk factors among a rural Yoruba southwestern Nigerian population: a population-based survey. Cardiovascular J Afr 21(1): 26-31.

3. Boutayeb A (2006) The double burden of communicable and non-communicable diseases in developing countries. Trans R Soc Trop Med Hyg 100(3): 191-199.

4. Mbanya JC, Motala AA, Sobngwi E, Assah FK, Enoru ST (2010) Diabetes in sub-Saharan Africa. Lancet 375(9733): 2254-2566.

5. Sobngwi E, Mauvais-Jarvis F, Vexiau P, Mbanya JC (2001) Diabetes in Africans. Part 1: epidemiology and clinical specificities. Diabetes Metab 27(6): 628-634.

6. Christensen DL, Friis H, Mwaniki DL, Kilonzo B, Tetens I, et al. (2009) Prevalence of glucose intolerance and associated risk factors in rural and urban populations of different ethnic groups in Kenya. Diabetes Res Clin Pract 84(3): 303-310.

7. Obirikorang C, Osakunor DN, Anto EO, Amponsah SO, Adarkwa OK (2015) Obesity and Cardio-Metabolic Risk Factors in an Urban and Rural Population in the Ashanti Region-Ghana: A Comparative Cross-Sectional Study. PLoS One 10(6): 0129494.

8. Re RN (2009) Obesity-related hypertension. Ochsner J 9(3): 133-136.

9. Carrère $\mathrm{P}$, Fagour C, Sportouch D, Gane-Troplent F, Hélène-Pelage J, et al. (2018) Diabetes mellitus and obesity in the French Caribbean: A special vulnerability for women?. Women Health 58(2): 145-159.

10. Nayak SB, Rahming V, Raghunanan Y, Raghoonath C, Rahman A, et al. (2016) Prevalence of Diabetes, Obesity and Dyslipidemia in Persons within High- and LowIncome Groups Living in North and South Trinidad. J Clin Diagn Res 10(5): 08-13.

11. Expert Panel on Detection, Evaluation, and Treatment of High Blood Cholesterol in Adults (2001) Executive Summary of the Third Report of the National Cholesterol Education Program (NCEP) Expert Panel on Detection, Evaluation, and Treatment of High Blood Cholesterol in Adults (Adult Treatment Panel III). JAMA 285(19): 24862497.

12. Danaei G, Finucane MM, Lu Y, Singh GM, Cowan MJ, et al. (2011) National, regional, and global trends in fasting plasma glucose and diabetes prevalence since 1980: systematic analysis of health examination surveys and epidemiological studies with 370 country-years and $2 \bullet 7$ million participants. Lancet 378(9785): 31-40.

13. Finucane MM, Stevens GA, Cowan MJ, Danaei G, Lin JK, et al. (2011) National, regional, and global trends in bodymass index since 1980: systematic analysis of health examination surveys and epidemiological studies with 
960 country-years and $9 \cdot 1$ million participants. Lancet 377: 557-567.

14. Wolf-Maier K, Cooper RS, Banegas JR, Giampaoli S, Hense HW, et al. (2003) Hypertension prevalence and blood pressure levels in 6 European countries, Canada, and the United States. JAMA 289(18): 2363-2369.

15. Hajjar I, Kotchen TA (2003) Trends in prevalence, awareness, treatment, and control of hypertension in the United States, 1988-2000. JAMA 290(2): 199-206.

16. Guo F, He D, Zhang W, Walton RG (2012) Trends in prevalence, awareness, management, and control of hypertension among United States adults, 1999 to 2010. J Am Coll Cardiol 60(7): 599-606.

17. Carroll MD, Lacher DA, Sorlie PD, Cleeman JI, Gordon DJ, et al. (2005) Trends in serum lipids and lipoproteins of adults, 1960-2002. JAMA 294(14): 1773-1781.

18. Tóth PP, Potter D, Ming EE (2012) Prevalence of lipid abnormalities in the United States: The National Health and Nutrition Examination Survey 2003-2006. J Clin Lipidol 6(4): 325-330.

19. Ford ES, Li C, Pearson WS, Zhao G, Mokdad AH (2010) Trends in hypercholesterolemia, treatment and control among United States adults. Int J Cardiol 140(2): 226235.

20. Weiwei C, Runlin G, Lisheng L, Manlu Z, Wen W, et al. (2016) Outline of the report on cardiovascular diseases in China, 2014. Eur Heart J Suppl 18(F): 2-11.

21. NCD Risk Factor Collaboration (NCD-RisC) (2016) Trends in adult body-mass index in 200 countries from 1975 to 2014: a pooled analysis of 1698 population-based measurement studies with $19 \cdot 2$ million participants. Lancet 387(10026): 1377-1396.

22. Ng SW, Howard AG, Wang HJ, Su C, Zhang B (2014) The physical activity transition among adults in China: 1991-

\section{Epidemiology International Journal}

2011. Obes Rev 15(1): 27-36.

23. Yao C, Wu Z, Wu Y (1993) The changing pattern of cardiovascular diseases in China. World Health Stat Q 46(2): 113-118.

24. Yusuf S, Reddy S, Ounpuu S, Anand S (2001) Global burden of cardiovascular diseases: Part II: variations in cardiovascular disease by specific ethnic groups and geographic regions and prevention strategies. Circulation 104(23): 2855-2864.

25. Egan BM, Zhao Y, Axon RN (2010) US trends in prevalence, awareness, treatment, and control of hypertension, 1988-2008. JAMA 303(20): 2043-2050.

26. Hata J, Ninomiya T, Hirakawa Y, Nagata M, Mukai N, et al. (2013) Secular trends in cardiovascular disease and its risk factors in Japanese: half-century data from the Hisayama Study (1961-2009). Circulation 128(11): 1198-1205.

27. Neuhauser HK, Adler C, Rosario AS, Diederichs C, Ellert U (2015) Hypertension prevalence, awareness, treatment and control in Germany 1998 and 2008-11. J Hum Hypertens 29(4): 247-253.

28. Di Lonardo A, Donfrancesco C, Palmieri L, Vanuzzo D, Giampaoli S(2017) Time Trends of High Blood Pressure Prevalence, Awareness and Control in the Italian General Population: Surveys of the National Institute of Health. High Blood Press Cardiovascular Prev 24(2): 193-200.

29. Kim HJ, Kim Y, Cho Y, Jun B, Oh KW (2014) Trends in the prevalence of major cardiovascular disease risk factors among Korean adults: results from the Korea National Health and Nutrition Examination Survey, 1998-2012. Int J Cardiol 174(1): 64-72.

30. Wang J, Zhang L, Wang F, Liu L, Wang H (2014) Prevalence, awareness, treatment, and control of hypertension in China: results from a national survey. Am J Hypertens 27(11): 1355-1361.



